\title{
Alternating iterative algorithms for the split equality problem without prior knowledge of operator norms
}

Hai $\mathrm{Yu}^{1 *}$ and Fenghui Wang ${ }^{1}$

${ }^{*}$ Correspondence: yuhai2000@126.com

1 Department of Mathematics, Luoyang Normal University,

Luoyang 471022, China

\section{Springer}

\begin{abstract}
In this paper, we study the alternating CQ algorithm for solving the split equality problem in Hilbert spaces. It is, however, not easy to implement since its selection of the stepsize requires prior information on the norms of bounded linear operators. To avoid this difficulty, we propose several modified algorithms in which the selection of the stepsize is independent of the norms. In particular, we consider the case whenever the convex sets involved are level sets of given convex functions.
\end{abstract}

MSC: 47J25; 47J20; 49N45; 65J15

Keywords: Split equality problem; Alternating CQ algorithm; Variable stepsize

\section{Introduction}

The split feasibility problem (SFP) requires finding a point $x \in H_{1}$ satisfying the property

$$
x \in C \text { and } A x \in Q \text {, }
$$

where $A: H_{1} \rightarrow H_{2}$ is a bounded linear operator, and $C$ and $Q$ are two nonempty closed and convex subsets of Hilbert spaces $H_{1}$ and $H_{2}$, respectively. The SFP was first introduced by Censor and Elfving [5] and has a very broad range of applications in many disciplines including signal processing, image reconstruction problem, and radiation therapy; see [2, $3,11]$. Various iterative algorithms have been constructed to solve SFP (1); see [10, 21-24, 26]. An iterative algorithm for solving the SFP, called the CQ algorithm, has the following iterative step:

$$
x_{n+1}=P_{C}\left(x_{n}-\gamma A^{*}\left(I-P_{Q}\right) A x_{n}\right),
$$

where $\gamma \in\left(0, \frac{2}{\|A\|^{2}}\right), I$ denotes the identity operator, $A^{*}$ denotes the adjoint of $A$, and $P_{C}$ and $P_{Q}$ are projections onto $C$ and $Q$, respectively. The SFP can be also solved by a different method [19, 29]:

$$
x_{n+1}=x_{n}-\gamma_{n}\left[\left(I-P_{C}\right) x_{n}+A^{*}\left(I-P_{Q}\right) A x_{n}\right],
$$

(c) The Author(s) 2020. This article is licensed under a Creative Commons Attribution 4.0 International License, which permits use, sharing, adaptation, distribution and reproduction in any medium or format, as long as you give appropriate credit to the original author(s) and the source, provide a link to the Creative Commons licence, and indicate if changes were made. The images or other third party material in this article are included in the article's Creative Commons licence, unless indicated otherwise in a credit line to the material. If material is not included in the article's Creative Commons licence and your intended use is not permitted by statutory regulation or exceeds the permitted use, you will need to obtain permission directly from the copyright holder. To view a copy of this licence, visit http://creativecommons.org/licenses/by/4.0/. 
where

$$
\gamma_{n}=\frac{\left\|\left(I-P_{C}\right) x_{n}\right\|^{2}+\left\|\left(I-P_{Q}\right) A x_{n}\right\|^{2}}{\left\|\left(I-P_{C}\right) x_{n}+A^{*}\left(I-P_{Q}\right) A x_{n}\right\|^{2}} .
$$

It is known that both (2) and (3) converge weakly to a solution of the SFP if it is consistent, that is, its solution set is nonempty.

Recently, Moudafi [13] introduced the split equality problem (SEP):

Find $x \in C, y \in Q$ such that $A x=B y$.

Here, $C \subseteq H_{1}, Q \subseteq H_{2}$ are two nonempty closed and convex subsets, and $A: H_{1} \rightarrow H_{3}$, $B: H_{2} \rightarrow H_{3}$ are two bounded linear operators, where $H_{1}, H_{2}, H_{3}$ are Hilbert spaces. In what follows, we always assume that the SEP is consistent, namely

$$
S=\{(x, y) \in C \times Q \mid A x=B y\} \neq \emptyset .
$$

Many algorithms for solving the SEP have been proposed; see $[8,9,12,13,15,20]$. In particular, Byrne and Moudafi $[4,14]$ introduced the simultaneous CQ algorithm:

$$
\left\{\begin{array}{l}
x_{n+1}=P_{C}\left(x_{n}-\gamma_{n} A^{*}\left(A x_{n}-B y_{n}\right)\right), \\
y_{n+1}=P_{Q}\left(y_{n}+\gamma_{n} B^{*}\left(A x_{n}-B y_{n}\right)\right),
\end{array}\right.
$$

where $\gamma_{n} \in\left(\epsilon, 2 /\left(\|A\|^{2}+\|B\|^{2}\right)-\epsilon\right)$ for small enough $\epsilon>0$. To determine stepsize $\gamma_{n}$ in (5), one needs first to calculate or estimate the norms $\|A\|$ and $\|B\|$, which is in general difficult or even impossible. To overcome this drawback, many authors have conducted worthwhile works[6-8, 19]. Among these works, Wang [19] suggested a novel variable-step:

$$
\tau_{n}=\frac{\rho_{n}}{\max \left(\left\|A^{*}\left(A x_{n}-B y_{n}\right)\right\|,\left\|B^{*}\left(A x_{n}-B y_{n}\right)\right\|\right)},
$$

where $\left\{\rho_{n}\right\}$ is a sequence of positive real numbers such that

$$
\sum_{n=0}^{\infty} \rho_{n}=\infty, \quad \sum_{n=0}^{\infty} \rho_{n}^{2}<\infty
$$

For solving the SEP, Moudafi [13] also introduced the alternating CQ-algorithm:

$$
\left\{\begin{array}{l}
x_{n+1}=P_{C}\left(x_{n}-\gamma_{n} A^{*}\left(A x_{n}-B y_{n}\right)\right), \\
y_{n+1}=P_{Q}\left(y_{n}+\gamma_{n} B^{*}\left(A x_{n+1}-B y_{n}\right)\right),
\end{array}\right.
$$

where $\left\{\gamma_{n}\right\}$ is a nondecreasing sequence such that $\gamma_{n} \in\left(\epsilon, \min \left(\frac{1}{\|A\|^{2}}, \frac{1}{\|B\|^{2}}\right)-\epsilon\right)$ for small enough $\epsilon>0$. It is worth noting that in algorithm (8) the choice of the stepsize still depends on the norms $\|A\|$ and $\|B\|$. Thus, a similar question arises: Does there exist a way to select the stepsize in algorithm (8) that does not depend on the operator norms? It is the purpose of this paper to answer this question affirmatively. Motivated by the choice of stepsize (6), we propose three alternating iterative algorithms for the SEP, in which the choice of the 
stepsize is independent of the norms $\|A\|$ and $\|B\|$. To the best of our knowledge, this is the first work to study alternating iterative algorithms for the SEP without prior knowledge of operator norms.

\section{Preliminaries}

Throughout this paper, we always assume that $H$ is a real Hilbert space with the inner product $\langle\cdot, \cdot\rangle$ and the norm $\|\cdot\|$, and $C$ is a nonempty closed convex subset in $H$. We denote by $I$ the identity operator on $H$, and by $\operatorname{Fix}(T)$ the set of the fixed points of an operator $T$. Given a sequence $\left\{x_{n}\right\}$ in $H, \omega_{w}\left(x_{n}\right)$ stands for the set of cluster points in the weak topology. The notation $\rightarrow$ stands for strong convergence and $\rightarrow$ stands for weak convergence.

Definition 2.1 Let $T: H \rightarrow H$ be an operator. Then $T$ is

(i) nonexpansive if

$$
\|T x-T y\| \leq\|x-y\|, \quad \forall x, y \in H
$$

(ii) firmly nonexpansive if

$$
\|T x-T y\|^{2} \leq\|x-y\|^{2}-\|(I-T) x-(I-T) y\|^{2}, \quad \forall x, y \in H .
$$

Definition 2.2 For any $x \in H$, the metric projection onto $C$ is defined as

$$
P_{C} x=\operatorname{argmin}\{\|y-x\| \mid y \in C\} .
$$

The projection $P_{C}$ has the following well-known properties.

Lemma $2.3([1,17])$ Let $x, y \in H$ and $z \in C$. Then

(i) $\left\langle x-P_{C} x, z-P_{C} x\right\rangle \leq 0$;

(ii) $P_{C}$ is firmly nonexpansive;

(iii) $\left\langle P_{C} x-P_{C} y, x-y\right\rangle \geq\left\|P_{C} x-P_{C} y\right\|^{2}$.

Definition 2.4 Let $T: H \rightarrow H$ be an operator with $\operatorname{Fix}(T) \neq \emptyset$. Then $I-T$ is said to be demiclosed at zero if, for any $\left\{x_{n}\right\}$ in $H$, the following implication holds:

$$
x_{n} \rightarrow x \quad \text { and } \quad(I-T) x_{n} \rightarrow 0 \quad \Longrightarrow \quad x \in \operatorname{Fix}(T) .
$$

It is well known that $I-T$ is demiclosed at zero if $T$ is nonexpansive $[1,17]$. Since $P_{C}$ is clearly nonexpansive, then $I-P_{C}$ is demiclosed at zero.

Definition 2.5 Let $\lambda \in(0,1)$ and $f: H \rightarrow(-\infty,+\infty]$ be a proper function.

(i) The function $f$ is convex if

$$
f(\lambda x+(1-\lambda) y) \leq \lambda f(x)+(1-\lambda) f(y), \quad \forall x, y \in H .
$$

(ii) A vector $u \in H$ is a subgradient of $f$ at a point $x$ if

$$
f(y) \geq f(x)+\langle u, y-x\rangle, \quad \forall y \in H .
$$


(iii) The set of all subgradients of $f$ at $x$, denoted by $\partial f(x)$, is called the subdifferential of $f$.

Next, we state the following lemmas which will be used in the sequel.

Lemma 2.6 ([1]) Let $f: H \rightarrow(-\infty,+\infty]$ be a proper convex function. Then $f$ is semicontinuous if and only if it is weakly semicontinuous.

Lemma 2.7 ([18]) Suppose that $\left\{a_{n}\right\}$ and $\left\{b_{n}\right\}$ are two sequences of nonnegative numbers such that

$$
a_{n+1} \leq a_{n}+b_{n}, \quad n \geq 0 .
$$

If $\sum_{n=0}^{\infty} b_{n}$ converges, then $\lim _{n \rightarrow \infty} a_{n}$ exists.

Lemma 2.8 ([25]) Let $\left\{x_{n}\right\}$ be a sequence in $H$ satisfying the properties:

(i) $\lim _{n \rightarrow \infty}\left\|x_{n}-x\right\|$ exists for each $x \in C$;

(ii) $\omega_{w}\left(x_{n}\right) \subseteq C$.

Then $\left\{x_{n}\right\}$ is weakly convergent to a point in $C$.

Lemma 2.9 Assume $\left\{a_{n}\right\},\left\{\gamma_{n}\right\},\left\{b_{n}\right\}$, and $\left\{c_{n}\right\}$ are sequences of nonnegative real numbers such that

$$
a_{n+1} \leq\left(1+\gamma_{n}\right)\left(a_{n}-b_{n}+c_{n}\right), \quad n \geq 0,
$$

where $\sum_{n=0}^{\infty} \gamma_{n}<\infty$ and $\sum_{n=0}^{\infty} c_{n}<\infty$. Then

(i) $\lim _{n \rightarrow \infty} a_{n}$ exists,

(ii) $\sum_{n=0}^{\infty} b_{n}<\infty$.

Proof (i) Since $\sum_{n=0}^{\infty} \gamma_{n}$ is convergent, we have $\prod_{n=0}^{\infty}\left(1+\gamma_{n}\right)$ is convergent. Let $\prod_{n=0}^{\infty}(1+$ $\left.\gamma_{n}\right)=\gamma$. It follows from (9) that

$$
\begin{aligned}
a_{n} & \leq\left(1+\gamma_{n-1}\right) a_{n-1}+\left(1+\gamma_{n-1}\right) c_{n-1} \\
& \leq\left(1+\gamma_{n-1}\right)\left(1+\gamma_{n-2}\right) a_{n-2}+\left(1+\gamma_{n-1}\right)\left(1+\gamma_{n-2}\right) c_{n-2}+\left(1+\gamma_{n-1}\right) c_{n-1} \\
& \leq \cdots \\
& \leq \prod_{k=0}^{n-1}\left(1+\gamma_{k}\right) a_{0}+\sum_{j=0}^{n-1}\left(\prod_{k=j}^{n-1}\left(1+\gamma_{k}\right) c_{j}\right) \\
& \leq \gamma a_{0}+\gamma \sum_{j=0}^{n-1} c_{j} \\
& \leq \gamma a_{0}+\gamma c
\end{aligned}
$$

where $c=\sum_{n=0}^{\infty} c_{n}$. This implies that

$$
a_{n+1} \leq\left(1+\gamma_{n}\right) a_{n}+\left(1+\gamma_{n}\right) c_{n}
$$




$$
\leq a_{n}+\gamma_{n}\left(\gamma a_{0}+\gamma c\right)+\gamma c_{n}
$$

Since $\sum_{n=0}^{\infty} \gamma_{n}<\infty$ and $\sum_{n=0}^{\infty} c_{n}<\infty$, by Lemma 2.7, part (i) holds.

(ii) It follows from (9) that

$$
\begin{aligned}
a_{n+1} \leq & \left(1+\gamma_{n}\right) a_{n}-\left(1+\gamma_{n}\right) b_{n}+\left(1+\gamma_{n}\right) c_{n} \\
\leq & \left(1+\gamma_{n}\right)\left(1+\gamma_{n-1}\right) a_{n-1}-\left(1+\gamma_{n}\right)\left(1+\gamma_{n-1}\right) b_{n-1}-\left(1+\gamma_{n}\right) b_{n} \\
& +\left(1+\gamma_{n}\right)\left(1+\gamma_{n-1}\right) c_{n-1}+\left(1+\gamma_{n}\right) c_{n} \\
\leq & \cdots \\
\leq & \prod_{k=0}^{n}\left(1+\gamma_{k}\right) a_{0}-\sum_{j=0}^{n}\left(\prod_{k=j}^{n}\left(1+\gamma_{k}\right) b_{j}\right)+\sum_{j=0}^{n}\left(\prod_{k=j}^{n}\left(1+\gamma_{k}\right) c_{j}\right) \\
\leq & \gamma a_{0}+\gamma c-\sum_{j=0}^{n} b_{j},
\end{aligned}
$$

where the last inequality holds since $\prod_{k=j}^{n}\left(1+\gamma_{k}\right) \geq 1$. So we obtain

$$
\sum_{j=0}^{n} b_{j} \leq \gamma a_{0}+\gamma c
$$

which means that $\sum_{n=0}^{\infty} b_{n}<\infty$.

Lemma 2.10 Let $\left\{u_{n}\right\},\left\{\rho_{n}\right\}$ be sequences of nonnegative real numbers such that

(i) $\sum_{k=0}^{\infty} \rho_{n} u_{n}<\infty$;

(ii) there exists some $M>0$ such that, for all $k \geq 0,\left|u_{n+1}-u_{n}\right| \leq M \rho_{n}$;

(iii) $\left\{\rho_{n}\right\}$ satisfies condition (7).

Then $\lim _{n \rightarrow \infty} u_{n}=0$.

Proof It follows from (i) and the assumption $\sum_{k=0}^{\infty} \rho_{n}=\infty$ that

$$
\liminf _{n \rightarrow \infty} u_{n}=0
$$

On the other hand, observe that

$$
\begin{aligned}
u_{n+1}^{2} & =u_{n}^{2}+2 u_{n}\left(u_{n+1}-u_{n}\right)+\left(u_{n+1}-u_{n}\right)^{2} \\
& \leq u_{n}^{2}+2 M \rho_{n} u_{n}+M^{2} \rho_{n}^{2} .
\end{aligned}
$$

It is clear that $\sum_{n=0}^{\infty}\left(2 M \rho_{n} u_{n}+M^{2} \rho_{n}^{2}\right)<\infty$ due to (i) and (7). Applying Lemma 2.7 to (10), we obtain that $\lim _{n \rightarrow \infty} u_{n}$ exists. Hence $\lim _{n \rightarrow \infty} u_{n}=0$, since we have shown that $\liminf _{n \rightarrow \infty} u_{n}=0$.

\section{Alternating iterative algorithm I}

As shown in the introduction, one needs first to calculate (or at least estimate) the norms $\|A\|$ and $\|B\|$ when algorithm (8) is implemented. But this is difficult or even impossible. To 
overcome this difficulty, we aim to introduce the following alternating iterative algorithm which does not depend on the norms. In this and next sections, we mainly consider the case whenever the projections $P_{C}$ and $P_{Q}$ have closed-form expressions; for example, half spaces and closed balls.

Algorithm 3.1 Let $\left(x_{0}, y_{0}\right) \in H_{1} \times H_{2}$ be arbitrary, $\delta>0$ be a constant, and $\left\{\rho_{n}\right\}$ be a sequence of positive real numbers. Given $\left(x_{n}, y_{n}\right)$, construct $\left(x_{n+1}, y_{n+1}\right)$ via the formula

$$
\left\{\begin{array}{l}
x_{n+1}=P_{C}\left(x_{n}-\tau_{n} A^{*}\left(A x_{n}-B y_{n}\right)\right), \\
y_{n+1}=P_{Q}\left(y_{n}+\tau_{n} B^{*}\left(A x_{n+1}-B y_{n}\right)\right),
\end{array}\right.
$$

where $\tau_{n}=\rho_{n}\left(\max \left\{\left\|A^{*}\left(A x_{n}-B y_{n}\right)\right\|,\left\|B^{*}\left(A x_{n}-B y_{n}\right)\right\|, \delta\right\}\right)^{-1}$.

Theorem 3.2 Let $\left\{\left(x_{n}, y_{n}\right)\right\}$ be the sequence generated by Algorithm 3.1. If $\left\{\rho_{n}\right\}$ satisfies condition (7), then $\left\{\left(x_{n}, y_{n}\right)\right\}$ converges weakly to a solution of SEP (4).

Proof Let $\left(x^{*}, y^{*}\right) \in S$ be arbitrarily chosen. Then $x^{*} \in C, y^{*} \in Q$ and $A x^{*}=B y^{*}$. Let

$$
z_{n}=P_{Q}\left(y_{n}+\tau_{n} B^{*}\left(A x_{n}-B y_{n}\right)\right)
$$

From Lemma 2.3 and the obvious fact that $x_{n} \in C$, it follows that

$$
\begin{aligned}
\left\|x_{n+1}-x_{n}\right\| & =\left\|P_{C}\left(x_{n}-\tau_{n} A^{*}\left(A x_{n}-B y_{n}\right)\right)-P_{C} x_{n}\right\| \\
& \leq \tau_{n}\left\|A^{*}\left(A x_{n}-B y_{n}\right)\right\| \leq \rho_{n},
\end{aligned}
$$

and

$$
\begin{aligned}
\left\|y_{n+1}-z_{n}\right\| & =\left\|P_{Q}\left(y_{n}+\tau_{n} B^{*}\left(A x_{n+1}-B y_{n}\right)\right)-P_{Q}\left(y_{n}+\tau_{n} B^{*}\left(A x_{n}-B y_{n}\right)\right)\right\| \\
& \leq\left\|\tau_{n} B^{*}\left(A x_{n+1}-A x_{n}\right)\right\| \\
& \leq \tau_{n}\|B\|\|A\|\left\|x_{n+1}-x_{n}\right\| \\
& \leq \rho_{n}^{2} \frac{1}{\delta}\|B\|\|A\| .
\end{aligned}
$$

On the other hand, in view of (11) and Lemma 2.3, we have

$$
\begin{aligned}
\left\|x_{n+1}-x^{*}\right\|^{2} & =\left\|P_{C}\left(x_{n}-\tau_{n} A^{*}\left(A x_{n}-B y_{n}\right)\right)-x^{*}\right\|^{2} \\
& \leq\left\|x_{n}-x^{*}\right\|^{2}-2 \tau_{n}\left\langle A^{*}\left(A x_{n}-B y_{n}\right), x_{n}-x^{*}\right\rangle+\tau_{n}^{2}\left\|A^{*}\left(A x_{n}-B y_{n}\right)\right\|^{2} \\
& \leq\left\|x_{n}-x^{*}\right\|^{2}-2 \tau_{n}\left\langle A x_{n}-B y_{n}, A x_{n}-A x^{*}\right\rangle+\rho_{n}^{2} .
\end{aligned}
$$

Similarly, we obtain

$$
\begin{aligned}
\left\|z_{n}-y^{*}\right\|^{2} & =\left\|P_{Q}\left(y_{n}+\tau_{n} B^{*}\left(A x_{n}-B y_{n}\right)\right)-y^{*}\right\|^{2} \\
& \leq\left\|y_{n}-y^{*}\right\|^{2}+2 \tau_{n}\left(B^{*}\left(A x_{n}-B y_{n}\right), y_{n}-y^{*}\right\rangle+\tau_{n}^{2}\left\|B^{*}\left(A x_{n}-B y_{n}\right)\right\|^{2}
\end{aligned}
$$




$$
\leq\left\|y_{n}-y^{*}\right\|^{2}+2 \tau_{n}\left|A x_{n}-B y_{n}, B y_{n}-B y^{*}\right\rangle+\rho_{n}^{2}
$$

By adding the last two inequalities and using the fact that $A x^{*}=B y^{*}$, we finally obtain

$$
\begin{aligned}
& \left\|x_{n+1}-x^{*}\right\|^{2}+\left\|z_{n}-y^{*}\right\|^{2} \\
& \quad \leq\left\|x_{n}-x^{*}\right\|^{2}+\left\|y_{n}-y^{*}\right\|^{2}-2 \tau_{n}\left\|A x_{n}-B y_{n}\right\|^{2}+2 \rho_{n}^{2} .
\end{aligned}
$$

It then follows from Young's inequality, (13), and (14) that

$$
\begin{aligned}
& \left\|x_{n+1}-x^{*}\right\|^{2}+\left\|y_{n+1}-y^{*}\right\|^{2} \\
& \quad=\left\|x_{n+1}-x^{*}\right\|^{2}+\left\|y_{n+1}-z_{n}+z_{n}-y^{*}\right\|^{2} \\
& \quad \leq\left\|x_{n+1}-x^{*}\right\|^{2}+\left(1+\rho_{n}^{2}\right)\left\|z_{n}-y^{*}\right\|^{2}+\left(1+\frac{1}{\rho_{n}^{2}}\right)\left\|y_{n+1}-z_{n}\right\|^{2} \\
& \quad \leq\left(1+\rho_{n}^{2}\right)\left(\left\|x_{n+1}-x^{*}\right\|^{2}+\left\|z_{n}-y^{*}\right\|^{2}+\rho_{n}^{2}\|A\|^{2}\|B\|^{2} \frac{1}{\delta^{2}}\right) \\
& \quad \leq\left(1+\rho_{n}^{2}\right)\left(\left\|x_{n}-x^{*}\right\|^{2}+\left\|y_{n}-y^{*}\right\|^{2}-2 \tau_{n}\left\|A x_{n}-B y_{n}\right\|^{2}+\rho_{n}^{2}\left(2+\|A\|^{2}\|B\|^{2} \frac{1}{\delta^{2}}\right)\right) .
\end{aligned}
$$

Now, by setting $a_{n}=\left\|x_{n}-x^{*}\right\|^{2}+\left\|y_{n}-y^{*}\right\|^{2}, \gamma_{n}=\rho_{n}^{2}, b_{n}=2 \tau_{n}\left\|A x_{n}-B y_{n}\right\|^{2}$, and $c_{n}=\rho_{n}^{2}(2+$ $\left.\|A\|^{2}\|B\|^{2} \frac{1}{\delta^{2}}\right)$, we obtain

$$
a_{n+1} \leq\left(1+\gamma_{n}\right)\left(a_{n}-b_{n}+c_{n}\right)
$$

Applying Lemma 2.9 to (15), we conclude that there exists $a^{*} \geq 0$ such that $\lim _{n \rightarrow \infty} a_{n}=a^{*}$ and

$$
\sum_{n=0}^{\infty} \tau_{n}\left\|A x_{n}-B y_{n}\right\|^{2}<\infty
$$

Hence $\left\{a_{n}\right\}$ is bounded, and so are $\left\{x_{n}\right\}$ and $\left\{y_{n}\right\}$. Since $A$ and $B$ are bounded linear operators, there exists $M_{1}>0$ such that $\left\|A^{*}\left(A x_{n}-B y_{n}\right)\right\| \leq M_{1},\left\|B^{*}\left(A x_{n}-B y_{n}\right)\right\| \leq M_{1}$, and $\left\|A x_{n+1}-B y_{n+1}\right\|+\left\|A x_{n}-B y_{n}\right\| \leq M_{1}$. So we have

$$
\sum_{n=0}^{\infty} \frac{\rho_{n}}{\max \left(M_{1}, \delta\right)}\left\|A x_{n}-B y_{n}\right\|^{2} \leq \sum_{n=0}^{\infty} \tau_{n}\left\|A x_{n}-B y_{n}\right\|^{2}<\infty .
$$

This implies that

$$
\sum_{n=0}^{\infty} \rho_{n}\left\|A x_{n}-B y_{n}\right\|^{2}<\infty
$$

We next show $\lim _{n \rightarrow \infty}\left\|A x_{n}-B y_{n}\right\|^{2}=0$. From (13) and the obvious fact that $y_{n} \in Q$, it follows that

$$
\begin{aligned}
\left\|y_{n+1}-y_{n}\right\| & \leq\left\|y_{n+1}-z_{n}\right\|+\left\|z_{n}-y_{n}\right\| \\
& =\left\|y_{n+1}-z_{n}\right\|+\left\|P_{Q}\left(y_{n}+\tau_{n} B^{*}\left(A x_{n}-B y_{n}\right)\right)-P_{Q} y_{n}\right\|
\end{aligned}
$$




$$
\begin{aligned}
& \leq \rho_{n}^{2} \frac{1}{\delta}\|B\|\|A\|+\tau_{n}\left\|B^{*}\left(A x_{n}-B y_{n}\right)\right\| \\
& \leq \rho_{n}^{2} \frac{1}{\delta}\|B\|\|A\|+\rho_{n} .
\end{aligned}
$$

Let $u_{n}=\left\|A x_{n}-B y_{n}\right\|^{2}$. By the last inequality and (12), we have

$$
\begin{aligned}
\left|u_{n+1}-u_{n}\right| & =\left|\left\|A x_{n+1}-B y_{n+1}\right\|-\left\|A x_{n}-B y_{n}\right\|\right|\left(\left\|A x_{n+1}-B y_{n+1}\right\|+\left\|A x_{n}-B y_{n}\right\|\right) \\
& \leq\left\|\left(A x_{n+1}-B y_{n+1}\right)-\left(A x_{n}-B y_{n}\right)\right\| M_{1} \\
& \leq\left(\|A\|\left\|x_{n+1}-x_{n}\right\|+\|B\|\left\|y_{n+1}-y_{n}\right\|\right) M_{1} \\
& \leq \rho_{n}\left(\|A\|+\|B\|+\rho_{n} \frac{1}{\delta}\|B\|^{2}\|A\|\right) M_{1} \\
& \leq \rho_{n} M_{2},
\end{aligned}
$$

where $M_{2}$ is a positive number such that $\left(\|A\|+\|B\|+\rho_{n} \frac{1}{\delta}\|B\|^{2}\|A\|\right) M_{1} \leq M_{2}$ for all $n \geq 0$. Therefore, by Lemma 2.10, we have $\lim _{n \rightarrow \infty}\left\|A x_{n}-B y_{n}\right\|^{2}=0$.

Now we turn to prove that $\omega_{w}\left(x_{n}, y_{n}\right) \subseteq S$. Let $\bar{x}$ and $\bar{y}$ be weak cluster points of the sequences $\left\{x_{n}\right\}$ and $\left\{y_{n}\right\}$, respectively. We assume that $x_{n_{k}} \rightarrow \bar{x}$ and $y_{n_{k}} \rightarrow \bar{y}$, where $\left\{x_{n_{k}}\right\}$ and $\left\{y_{n_{k}}\right\}$ are subsequences of $\left\{x_{n}\right\}$ and $\left\{y_{n}\right\}$, respectively. Since $\left\{x_{n_{k}}\right\} \subseteq C,\left\{y_{n_{k}}\right\} \subseteq Q$ and $C$ and $Q$ are closed and convex, we have $\bar{x} \in C$ and $\bar{y} \in Q$. Furthermore, the weak convergence of $\left\{A x_{n_{k}}-B y_{n_{k}}\right\}$ to $A \bar{x}-B \bar{y}$ and the weakly lower semicontinuity of the squared norm imply

$$
\|A \bar{x}-B \bar{y}\|^{2} \leq \liminf _{k \rightarrow \infty}\left\|A x_{n_{k}}-B y_{n_{k}}\right\|^{2}=0 .
$$

Hence $(\bar{x}, \bar{y}) \in S$.

It is readily seen that $\lim _{n \rightarrow \infty}\left\|x_{n}-x^{*}\right\|^{2}+\left\|y_{n}-y^{*}\right\|^{2}$ exists for each $\left(x^{*}, y^{*}\right) \in S$ and $\omega_{w}\left(x_{n}, y_{n}\right) \subseteq S$. Therefore, it follows from Lemma 2.8 that $\left\{\left(x_{n}, y_{n}\right)\right\}$ converges weakly to a solution of SEP (4).

Remark 3.3 It is clear that our choice of the stepsize in Algorithm 3.1 does not need any information on the values of $\|A\|$ and $\|B\|$.

\section{Alternating iterative algorithm II}

In this section, we propose another alternating iterative algorithm for problem (4), in which the choice of the stepsize does not need any prior information of operator norms.

Algorithm 4.1 Let $\left(x_{0}, y_{0}\right) \in H_{1} \times H_{2}$ be arbitrary, $\delta>0$ be a constant, and $\left\{\rho_{n}\right\}$ be a sequence of positive real numbers. Given $\left(x_{n}, y_{n}\right)$, construct $\left(x_{n+1}, y_{n+1}\right)$ via the formula

$$
\left\{\begin{array}{l}
x_{n+1}=x_{n}-\tau_{n}\left[\left(I-P_{C}\right) x_{n}+A^{*}\left(A x_{n}-B y_{n}\right)\right], \\
y_{n+1}=y_{n}-\tau_{n}\left[\left(I-P_{Q}\right) y_{n}-B^{*}\left(A x_{n+1}-B y_{n}\right)\right],
\end{array}\right.
$$

where $\tau_{n}=\rho_{n}\left(\max \left\{\left\|\left(I-P_{C}\right) x_{n}+A^{*}\left(A x_{n}-B y_{n}\right)\right\|,\left\|\left(I-P_{Q}\right) y_{n}-B^{*}\left(A x_{n}-B y_{n}\right)\right\|, \delta\right\}\right)^{-1}$.

Theorem 4.2 Let $\left\{\left(x_{n}, y_{n}\right)\right\}$ be the sequence generated by Algorithm 4.1. If $\left\{\rho_{n}\right\}$ satisfies condition (7), then $\left\{\left(x_{n}, y_{n}\right)\right\}$ converges weakly to a solution of SEP (4). 
Proof Let $\left(x^{*}, y^{*}\right) \in S$ be arbitrarily chosen. Then $x^{*} \in C, y^{*} \in Q$, and $A x^{*}=B y^{*}$. Let

$$
z_{n}=y_{n}-\tau_{n}\left[\left(I-P_{Q}\right) y_{n}-B^{*}\left(A x_{n}-B y_{n}\right)\right]
$$

Then we have

$$
\begin{aligned}
\left\|y_{n+1}-z_{n}\right\|^{2} & =\left\|\tau_{n} B^{*}\left(A x_{n+1}-A x_{n}\right)\right\|^{2} \\
& \leq \tau_{n}^{2}\|B\|^{2}\|A\|^{2}\left\|x_{n+1}-x_{n}\right\|^{2} \\
& \leq \tau_{n}^{2}\|B\|^{2}\|A\|^{2} \rho_{n}^{2} \\
& \leq \rho_{n}^{4} \frac{1}{\delta^{2}}\|B\|^{2}\|A\|^{2} .
\end{aligned}
$$

It follows from (16) that

$$
\begin{aligned}
\left\|x_{n+1}-x^{*}\right\|^{2}= & \left\|x_{n}-x^{*}\right\|^{2}-2 \tau_{n}\left\langle\left(I-P_{C}\right) x_{n}+A^{*}\left(A x_{n}-B y_{n}\right), x_{n}-x^{*}\right\rangle \\
& +\tau_{n}^{2}\left\|\left(I-P_{C}\right) x_{n}+A^{*}\left(A x_{n}-B y_{n}\right)\right\|^{2} \\
\leq & \left\|x_{n}-x^{*}\right\|^{2}-2 \tau_{n}\left\langle\left(I-P_{C}\right) x_{n}, x_{n}-x^{*}\right\rangle-2 \tau_{n}\left\langle A x_{n}-B y_{n}, A x_{n}-A x^{*}\right\rangle \\
& +\tau_{n}^{2}\left\|\left(I-P_{C}\right) x_{n}+A^{*}\left(A x_{n}-B y_{n}\right)\right\|^{2} \\
\leq & \left\|x_{n}-x^{*}\right\|^{2}-2 \tau_{n}\left\|\left(I-P_{C}\right) x_{n}\right\|^{2}-2 \tau_{n}\left\langle A x_{n}-B y_{n}, A x_{n}-A x^{*}\right\rangle+\rho_{n}^{2} .
\end{aligned}
$$

Similarly, we obtain

$$
\begin{aligned}
\left\|z_{n}-y^{*}\right\|^{2}= & \left\|y_{n}-y^{*}\right\|^{2}-2 \tau_{n}\left\langle\left(I-P_{Q}\right) y_{n}-B^{*}\left(A x_{n}-B y_{n}\right), y_{n}-y^{*}\right\rangle \\
& +\tau_{n}^{2}\left\|\left(I-P_{Q}\right) y_{n}-B^{*}\left(A x_{n}-B y_{n}\right)\right\|^{2} \\
\leq & \left\|y_{n}-y^{*}\right\|^{2}-2 \tau_{n}\left\langle\left(I-P_{Q}\right) y_{n}, y_{n}-y^{*}\right\rangle+2 \tau_{n}\left\langle A x_{n}-B y_{n}, B y_{n}-B y^{*}\right\rangle \\
& +\tau_{n}^{2}\left\|\left(I-P_{Q}\right) y_{n}-B^{*}\left(A x_{n}-B y_{n}\right)\right\|^{2} \\
\leq & \left\|y_{n}-y^{*}\right\|^{2}-2 \tau_{n}\left\|\left(I-P_{Q}\right) y_{n}\right\|^{2}+2 \tau_{n}\left\langle A x_{n}-B y_{n}, B y_{n}-B y^{*}\right\rangle+\rho_{n}^{2} .
\end{aligned}
$$

By adding the last two inequalities and using the fact $A x^{*}=B y^{*}$, we finally obtain

$$
\begin{aligned}
\left\|x_{n+1}-x^{*}\right\|^{2}+\left\|z_{n}-y^{*}\right\|^{2} \leq & \left\|x_{n}-x^{*}\right\|^{2}+\left\|y_{n}-y^{*}\right\|^{2}-2 \tau_{n}\left(\left\|\left(I-P_{C}\right) x_{n}\right\|^{2}\right. \\
& \left.+\left\|\left(I-P_{Q}\right) y_{n}\right\|^{2}+\left\|A x_{n}-B y_{n}\right\|^{2}\right)+2 \rho_{n}^{2} .
\end{aligned}
$$

It then follows from Young's inequality, (17), and (18) that

$$
\begin{aligned}
& \left\|x_{n+1}-x^{*}\right\|^{2}+\left\|y_{n+1}-y^{*}\right\|^{2} \\
& \quad=\left\|x_{n+1}-x^{*}\right\|^{2}+\left\|y_{n+1}-z_{n}+z_{n}-y^{*}\right\|^{2} \\
& \leq\left\|x_{n+1}-x^{*}\right\|^{2}+\left(1+\rho_{n}^{2}\right)\left\|z_{n}-y^{*}\right\|^{2}+\left(1+\frac{1}{\rho_{n}^{2}}\right)\left\|y_{n+1}-z_{n}\right\|^{2} \\
& \quad \leq\left(1+\rho_{n}^{2}\right)\left(\left\|x_{n+1}-x^{*}\right\|^{2}+\left\|z_{n}-y^{*}\right\|^{2}+\rho_{n}^{2} \frac{1}{\delta^{2}}\|B\|^{2}\|A\|^{2}\right)
\end{aligned}
$$




$$
\begin{aligned}
\leq & \left(1+\rho_{n}^{2}\right)\left[\left\|x_{n}-x^{*}\right\|^{2}+\left\|y_{n}-y^{*}\right\|^{2}-2 \tau_{n}\left(\left\|\left(I-P_{C}\right) x_{n}\right\|^{2}\right.\right. \\
& \left.\left.+\left\|\left(I-P_{Q}\right) y_{n}\right\|^{2}+\left\|A x_{n}-B y_{n}\right\|^{2}\right)+\rho_{n}^{2}\left(2+\frac{1}{\delta^{2}}\|B\|^{2}\|A\|^{2}\right)\right] .
\end{aligned}
$$

Now, by setting $a_{n}=\left\|x_{n}-x^{*}\right\|^{2}+\left\|y_{n}-y^{*}\right\|^{2}, \gamma_{n}=\rho_{n}^{2}, c_{n}=\rho_{n}^{2}\left(2+\frac{1}{\delta^{2}}\|B\|^{2}\|A\|^{2}\right)$ and

$$
b_{n}=2 \tau_{n}\left(\left\|\left(I-P_{C}\right) x_{n}\right\|^{2}+\left\|\left(I-P_{Q}\right) y_{n}\right\|^{2}+\left\|A x_{n}-B y_{n}\right\|^{2}\right),
$$

we can immediately obtain

$$
a_{n+1} \leq\left(1+\gamma_{n}\right)\left(a_{n}-b_{n}+c_{n}\right)
$$

Applying Lemma 2.9 to (19), we conclude that $\lim _{n \rightarrow \infty} a_{n}$ exists and

$$
\sum_{n=0}^{\infty} 2 \tau_{n}\left(\left\|\left(I-P_{C}\right) x_{n}\right\|^{2}+\left\|\left(I-P_{Q}\right) y_{n}\right\|^{2}+\left\|A x_{n}-B y_{n}\right\|^{2}\right)<\infty
$$

Therefore $\left\{a_{n}\right\}$ is bounded, and so are $\left\{x_{n}\right\}$ and $\left\{y_{n}\right\}$. Observe that

$$
\left\|P_{C} x_{n}-x^{*}\right\| \leq\left\|x_{n}-x^{*}\right\|, \quad\left\|P_{Q} y_{n}-y^{*}\right\| \leq\left\|y_{n}-y^{*}\right\| .
$$

This implies that $\left\{\left(I-P_{C}\right) x_{n}\right\}$ and $\left\{\left(I-P_{Q}\right) y_{n}\right\}$ are also bounded. Since $A$ and $B$ are bounded linear operators, there exists $M_{3}>0$ such that, for all $n \geq 0,\left\|\left(I-P_{C}\right) x_{n}+A^{*}\left(A x_{n}-B y_{n}\right)\right\| \leq$ $M_{3},\left\|\left(I-P_{Q}\right) y_{n}-B^{*}\left(A x_{n}-B y_{n}\right)\right\| \leq M_{3},\left\|\left(I-P_{C}\right) x_{n+1}\right\|+\left\|\left(I-P_{C}\right) x_{n}\right\|<M_{3}$, and $\|(I-$ $\left.P_{Q}\right) y_{n+1}\|+\|\left(I-P_{Q}\right) y_{n} \|<M_{3}$. Thus, we have

$$
\begin{aligned}
& \sum_{n=0}^{\infty} \frac{\rho_{n}}{\max \left(M_{3}, \delta\right)}\left(\left\|\left(I-P_{C}\right) x_{n}\right\|^{2}+\left\|\left(I-P_{Q}\right) y_{n}\right\|^{2}+\left\|A x_{n}-B y_{n}\right\|^{2}\right) \\
& \quad \leq \sum_{n=0}^{\infty} \tau_{n}\left(\left\|\left(I-P_{C}\right) x_{n}\right\|^{2}+\left\|\left(I-P_{Q}\right) y_{n}\right\|^{2}+\left\|A x_{n}-B y_{n}\right\|^{2}\right)<\infty .
\end{aligned}
$$

This implies that

$$
\sum_{n=0}^{\infty} \rho_{n}\left\|\left(I-P_{C}\right) x_{n}\right\|^{2}<\infty, \quad \sum_{n=0}^{\infty} \rho_{n}\left\|\left(I-P_{Q}\right) y_{n}\right\|^{2}<\infty,
$$

and

$$
\sum_{n=0}^{\infty} \rho_{n}\left\|A x_{n}-B y_{n}\right\|^{2}<\infty
$$

We next prove that $\lim _{n \rightarrow \infty}\left\|\left(I-P_{C}\right) x_{n}\right\|^{2}=0, \lim _{n \rightarrow \infty}\left\|\left(I-P_{Q}\right) y_{n}\right\|^{2}=0$, and $\lim _{n \rightarrow \infty}\left\|A x_{n}-B y_{n}\right\|^{2}=0$. From (17), we get

$$
\left\|y_{n+1}-y_{n}\right\| \leq\left\|y_{n+1}-z_{n}\right\|+\left\|z_{n}-y_{n}\right\| \leq \rho_{n}^{2} \frac{1}{\delta}\|B\|\|A\|+\rho_{n} .
$$


Observe that

$$
\begin{aligned}
& \left|\left\|\left(I-P_{C}\right) x_{n+1}\right\|^{2}-\left\|\left(I-P_{C}\right) x_{n}\right\|^{2}\right| \\
& \quad=\left|\left\|\left(I-P_{C}\right) x_{n+1}\right\|-\left\|\left(I-P_{C}\right) x_{n}\right\|\right|\left(\left\|\left(I-P_{C}\right) x_{n+1}\right\|+\left\|\left(I-P_{C}\right) x_{n}\right\|\right) \\
& \quad \leq\left\|\left(I-P_{C}\right) x_{n+1}-\left(I-P_{C}\right) x_{n}\right\| M_{3} \\
& \quad \leq\left\|x_{n+1}-x_{n}\right\| M_{3} \\
& \quad \leq M_{3} \rho_{n} .
\end{aligned}
$$

This along with (20) and Lemma 2.10 implies that $\lim _{n \rightarrow \infty}\left\|\left(I-P_{C}\right) x_{n}\right\|^{2}=0$. Similarly, we have

$$
\begin{aligned}
& \left|\left\|\left(I-P_{Q}\right) y_{n+1}\right\|^{2}-\left\|\left(I-P_{Q}\right) y_{n}\right\|^{2}\right| \\
& \quad=\left|\left\|\left(I-P_{Q}\right) y_{n+1}\right\|-\left\|\left(I-P_{Q}\right) y_{n}\right\|\right|\left(\left\|\left(I-P_{Q}\right) y_{n+1}\right\|+\left\|\left(I-P_{Q}\right) y_{n}\right\|\right) \\
& \quad \leq\left\|\left(I-P_{Q}\right) y_{n+1}-\left(I-P_{Q}\right) y_{n}\right\| M_{3} \\
& \quad \leq\left\|y_{n+1}-y_{n}\right\| M_{3} \\
& \quad \leq \rho_{n}\left(\rho_{n} \frac{1}{\delta}\|B\|\|A\|+1\right) M_{3} \\
& \quad \leq M_{4} \rho_{n},
\end{aligned}
$$

where $M_{4}$ is a positive number such that $\left(\rho_{n} \frac{1}{\delta}\|B\|\|A\|+1\right) M_{3} \leq M_{4}$ for all $n \geq 0$. Hence, by Lemma 2.10, we conclude that $\lim _{n \rightarrow \infty}\left\|\left(I-P_{Q}\right) y_{n}\right\|^{2}=0$. Using a similar method in Theorem 3.2, we obtain $\lim _{n \rightarrow \infty}\left\|A x_{n}-B y_{n}\right\|^{2}=0$.

Now, we show that $\omega_{w}\left(x_{n}, y_{n}\right) \subseteq S$. Let $\bar{x}$ and $\bar{y}$ be weak cluster points of the sequences $\left\{x_{n}\right\}$ and $\left\{y_{n}\right\}$, respectively. We assume that $\left\{x_{n_{k}}\right\}$ and $\left\{y_{n_{k}}\right\}$ are subsequences of $\left\{x_{n}\right\}$ and $\left\{y_{n}\right\}$ such that $x_{n_{k}} \rightarrow \bar{x}$ and $y_{n_{k}} \rightarrow \bar{y}$, respectively. Since $I-P_{C}$ and $I-P_{Q}$ are demiclosed at zero, it follows from $\left(I-P_{C}\right) x_{n_{k}} \rightarrow 0$ and $\left(I-P_{Q}\right) y_{n_{k}} \rightarrow 0$ that $\bar{x} \in C$ and $\bar{y} \in Q$. Furthermore, the weak convergence of $\left\{A x_{n_{k}}-B y_{n_{k}}\right\}$ to $A \bar{x}-B \bar{y}$ and the weakly lower semicontinuity of the squared norm imply

$$
\|A \bar{x}-B \bar{y}\|^{2} \leq \liminf _{k \rightarrow \infty}\left\|A x_{n_{k}}-B y_{n_{k}}\right\|^{2}=0 .
$$

Hence $(\bar{x}, \bar{y}) \in S$. In summary, we have proved that $\lim _{n \rightarrow \infty}\left\|x_{n}-x^{*}\right\|^{2}+\left\|y_{n}-y^{*}\right\|^{2}$ exists for each $\left(x^{*}, y^{*}\right) \in S$ and $\omega_{w}\left(x_{n}, y_{n}\right) \subseteq S$. Thus, we conclude from Lemma 2.8 that $\left\{\left(x_{n}, y_{n}\right)\right\}$ converges weakly to a solution of SEP (4).

\section{A relaxed alternating iterative algorithm}

In this section, we consider the case whenever $P_{C}$ or $P_{Q}$ fails to have a closed-form expression. Indeed, Moudafi [12] considered one of such cases when $C$ and $Q$ are level sets:

$$
C=\left\{x \in H_{1} \mid c(x) \leq 0\right\}
$$

and

$$
Q=\left\{y \in H_{2} \mid q(y) \leq 0\right\},
$$


where $c: H_{1} \rightarrow \mathbb{R}$ and $q: H_{2} \rightarrow \mathbb{R}$ are two convex lower semicontinuous and subdifferential functions on $H_{1}$ and $H_{2}$, respectively. Here the subdifferential operators $\partial c$ and $\partial q$ of $c$ and $q$ are assumed to be bounded, i.e., bounded on bounded sets. In this case, it is known that the associated projections are very hard to calculate. To overcome this difficulty, Moudafi [12] presented the relaxed alternating CQ-algorithm (RACQA):

$$
\left\{\begin{array}{l}
x_{n+1}=P_{C_{n}}\left(x_{n}-\gamma A^{*}\left(A x_{n}-B y_{n}\right)\right), \\
y_{n+1}=P_{Q_{n}}\left(y_{n}+\gamma B^{*}\left(A x_{n+1}-B y_{n}\right)\right),
\end{array}\right.
$$

where $\left\{C_{n}\right\}$ and $\left\{Q_{n}\right\}$ are two sequences of closed convex sets defined by

$$
C_{n}=\left\{x \in H_{1} \mid c\left(x_{n}\right)+\left\langle\xi_{n}, x-x_{n}\right\rangle \leq 0\right\}, \quad \xi_{n} \in \partial c\left(x_{n}\right)
$$

and

$$
Q_{n}=\left\{y \in H_{2} \mid q\left(y_{n}\right)+\left\langle\eta_{n}, y-y_{n}\right\rangle \leq 0\right\}, \quad \eta_{n} \in \partial q\left(y_{n}\right) .
$$

Since $C_{n}$ and $Q_{n}$ are clearly half-spaces, the associated projections thus have closed form expressions. This indicates that the implementation of RACQA is very easy. Under suitable conditions, Moudafi [12] proved that the RACQA converges weakly to a solution of (4).

Following the RACQA and our proposed algorithms, we now present a relaxed alternating iterative algorithm in which we just need projections onto half-spaces [16, 27, 28].

In what follows, we will treat SEP (4) under the following assumptions.

(A1) The sets $C$ and $Q$ are given in (21) and (22), respectively.

(A2) For any $x \in H_{1}$ and $y \in H_{2}$, at least one subgradient $\xi \in \partial c(x)$ and $\eta \in \partial q(y)$ can be calculated.

Remark 5.1 It follows from Lemma 2.6 that both $c$ and $q$ are weakly lower semicontinuous by condition (A2), since $c: H_{1} \rightarrow \mathbb{R}$ and $q: H_{2} \rightarrow \mathbb{R}$ are convex.

We are now in a position to present a relaxed alternative iterative algorithm that does not depend on operator norms for solving SEP (4).

Algorithm 5.2 Let $\left(x_{0}, y_{0}\right)$ be arbitrary, $\delta>0$ be a constant, and $\left\{\rho_{n}\right\}$ be a sequence of positive real numbers. Given $\left(x_{n}, y_{n}\right)$, construct $\left(x_{n+1}, y_{n+1}\right)$ via the formula

$$
\left\{\begin{array}{l}
x_{n+1}=x_{n}-\tau_{n}\left[\left(I-P_{C_{n}}\right) x_{n}+A^{*}\left(A x_{n}-B y_{n}\right)\right], \\
y_{n+1}=y_{n}-\tau_{n}\left[\left(I-P_{Q_{n}}\right) y_{n}-B^{*}\left(A x_{n+1}-B y_{n}\right)\right],
\end{array}\right.
$$

where $C_{n}$ and $Q_{n}$ are given as in (24) and (25), respectively, and

$$
\tau_{n}=\rho_{n}\left(\max \left\{\left\|\left(I-P_{C_{n}}\right) x_{n}+A^{*}\left(A x_{n}-B y_{n}\right)\right\|,\left\|\left(I-P_{Q_{n}}\right) y_{n}-B^{*}\left(A x_{n}-B y_{n}\right)\right\|, \delta\right\}\right)^{-1} .
$$

Remark 5.3 By the definition of the subgradient, it is obvious that $C \subseteq C_{n}$ and $Q \subseteq Q_{n}$ for all $n \geq 0$. Since $C_{n}$ and $Q_{n}$ are both half-spaces, the projections onto $C_{n}$ and $Q_{n}$ can be directly calculated. Thus Algorithm 5.2 is easily implementable. 
Theorem 5.4 Let $\left\{\left(x_{n}, y_{n}\right)\right\}$ be the sequence generated by Algorithm 5.2. If $\left\{\rho_{n}\right\}$ satisfies condition (7), then $\left\{\left(x_{n}, y_{n}\right)\right\}$ converges weakly to a solution of SEP (4).

Proof Take $\left(x^{*}, y^{*}\right) \in S$, i.e., $x^{*} \in C$ (and thus $\left.x^{*} \in C_{n}\right) ; y^{*} \in Q$ (and thus $y^{*} \in Q_{n}$ ), $A x^{*}=B y^{*}$. Similarly as in the proof of Theorem 4.2, we can conclude that $\lim _{n \rightarrow \infty}\left(\left\|x_{n}-x^{*}\right\|^{2}+\| y_{n}-\right.$ $\left.y^{*} \|^{2}\right)$ exists, $\lim _{n \rightarrow \infty}\left\|\left(I-P_{C_{n}}\right) x_{n}\right\|^{2}=0, \lim _{n \rightarrow \infty}\left\|\left(I-P_{Q_{n}}\right) y_{n}\right\|^{2}=0$, and $\lim _{n \rightarrow \infty} \| A x_{n}-$ $B y_{n} \|^{2}=0$.

We next show that $\omega_{w}\left(x_{n}, y_{n}\right) \subseteq S$. Since $\lim _{n \rightarrow \infty}\left(\left\|x_{n}-x^{*}\right\|^{2}+\left\|y_{n}-y^{*}\right\|^{2}\right)$ exists, the sequences $\left\{x_{n}\right\}$ and $\left\{y_{n}\right\}$ are bounded. Let $\bar{x}$ and $\bar{y}$ be weak cluster points of the sequences $\left\{x_{n}\right\}$ and $\left\{y_{n}\right\}$, respectively. Without loss of generality, we assume that $x_{n} \rightarrow \bar{x}$ and $y_{n} \rightarrow \bar{y}$. Since $\partial c$ is bounded on bounded sets, there is a constant $\sigma_{1}>0$ such that $\left\|\xi_{n}\right\| \leq \sigma_{1}$ for all $n \geq 0$. From (24) and the fact that $P_{C_{n}}\left(x_{n}\right) \in C_{n}$, it follows that

$$
c\left(x_{n}\right) \leq\left\langle\xi_{n}, x_{n}-P_{C_{n}} x_{n}\right\rangle \leq \sigma_{1}\left\|\left(I-P_{C_{n}}\right) x_{n}\right\| .
$$

The weakly lower semicontinuity of $c$ leads to

$$
c(\bar{x}) \leq \liminf _{n \rightarrow+\infty} c\left(x_{n}\right) \leq \sigma_{1} \liminf _{n \rightarrow+\infty}\left\|\left(I-P_{C_{n}}\right) x_{n}\right\|=0,
$$

and therefore $\bar{x} \in C$. Likewise, since $\partial q$ is bounded on bounded sets, there is a constant $\sigma_{2}>0$ such that $\left\|\eta_{n}\right\| \leq \sigma_{2}$ for all $n \geq 0$. From (25) and the fact that $P_{Q_{n}}\left(y_{n}\right) \in Q_{n}$, it follows that

$$
q\left(y_{n}\right) \leq\left\langle\eta_{n}, y_{n}-P_{Q_{n}} y_{n}\right\rangle \leq \sigma_{2}\left\|\left(I-P_{Q_{n}}\right) y_{n}\right\| .
$$

Again, the weakly lower semicontinuity of $q$ leads to

$$
q(\bar{y}) \leq \liminf _{n \rightarrow+\infty} q\left(y_{n}\right) \leq \sigma_{2} \liminf _{n \rightarrow+\infty}\left\|\left(I-P_{Q_{n}}\right) y_{n}\right\|=0,
$$

and therefore $\bar{y} \in Q$. Furthermore, the weak convergence of $\left\{A x_{n}-B y_{n}\right\}$ to $A \bar{x}-B \bar{y}$ and the weakly lower semicontinuity of the squared norm imply

$$
\|A \bar{x}-B \bar{y}\|^{2} \leq \liminf _{n \rightarrow+\infty}\left\|A x_{n}-B y_{n}\right\|^{2}=0 .
$$

Hence $(\bar{x}, \bar{y}) \in S$.

Finally, we deduce from Lemma 2.8 that $\left\{\left(x_{n}, y_{n}\right)\right\}$ converges weakly to a solution of SEP (4), since $\lim _{n \rightarrow \infty}\left\|x_{n}-x^{*}\right\|^{2}+\left\|y_{n}-y^{*}\right\|^{2}$ exists for each $\left(x^{*}, y^{*}\right) \in S$ and $\omega_{w}\left(x_{n}, y_{n}\right) \subseteq S$.

\section{Acknowledgements}

Not applicable.

\section{Funding}

This work was supported by the National Natural Science Foundation of China (Nos. 11971216, 11571005) and the

Foundation of He'nan Educational Committee (No. 20A110029).

Availability of data and materials

Not applicable. 
Authors' contributions

All authors contributed equally to the writing of this paper. All authors read and approved the final manuscript.

\section{Publisher's Note}

Springer Nature remains neutral with regard to jurisdictional claims in published maps and institutional affiliations.

Received: 30 June 2020 Accepted: 17 August 2020 Published online: 27 August 2020

\section{References}

1. Bauschke, H.H., Combettes, P.L.: Convex Analysis and Monotone Operator Theory in Hilbert Space. Springer, Berlin (2011)

2. Byrne, C.: Iterative oblique projection onto convex sets and the split feasibility problem. Inverse Probl. 18, 441-453 (2002)

3. Byrne, C.: A unified treatment of some iterative algorithms in signal processing and image reconstruction. Inverse Probl. 20, 103-120 (2004)

4. Byrne, C., Moudafi, A.: Extensions of the CQ algorithm for the split feasibility and split equality problems. J. Nonlinear Convex Anal. 18, 1485-1496 (2017)

5. Censor, Y., Elfving, T.: A multiprojection algorithm using Bregman projections in a product space. Numer. Algorithms 8, 221-239 (1994)

6. Chuang, C., Du, W.: Hybrid simultaneous algorithms for the split equality problem with applications. J. Inequal. Appl. 2016, 198 (2016)

7. Dong, Q., He, S.: Self-adaptive projection algorithms for solving the split equality problems. Fixed Point Theory 18(1), 191-202 (2017)

8. Dong, Q., He, S., Zhao, J.: Solving the split equality problem without prior knowledge of operator norms. Optimization 64(9), 1887-1906 (2015)

9. Dong, Q., Jiang, D.: Simultaneous and semi-alternating projection algorithms for solving split equality problems. J. Inequal. Appl. 2018, 4 (2018)

10. He, S., Tian, H., Xu, H.: The selective projection method for convex feasibility and split feasibility problems. J. Nonlinear Convex Anal. 19(7), 1199-1215 (2018)

11. López, G., Martín, V., Wang, F., Xu, H.K.: Solving the split feasibility problem without prior knowledge of matrix norms. Inverse Probl. 28, 085004 (2012)

12. Moudafi, A.: A relaxed alternating CQ-algorithm for convex feasibility problems. Nonlinear Anal. 79, 117-121 (2013)

13. Moudafi, A.: Alternating CQ-algorithm for convex feasibility and split fixed-point problems. J. Nonlinear Convex Anal. $15,809-818(2014)$

14. Moudafi, A., Al-Shemas, E.: Simultaneous iterative methods for split equality problems and applications. Trans. Math Program. Appl. 1, 1-11 (2013)

15. Naraghirad, E.: On an open question of Moudafi for convex feasibility problem in Hilbert spaces. Taiwan. J. Math. 18(2), 371-408 (2014)

16. Qu, B., Xiu, N.H.: A new halfspace-relaxation projection method for the split feasibility problem. Linear Algebra Appl. 428(5), 1218-1229 (2008)

17. Takahashi, W.: Nonlinear Functional Analysis, Fixed Point Theory and Its Applications. Yokahama Publishers, Yokahama (2000)

18. Tan, K.K., Xu, H.K.: Approximating fixed points of nonexpansive mappings by the Ishikawa iteration process. J. Math. Anal. Appl. 178, 301-308 (1993)

19. Wang, F:: A new iterative method for the split common fixed point problem in Hilbert spaces. Optimization 66 407-415 (2017)

20. Wang, F: On the convergence of $C Q$ algorithm with variable steps for the split equality problem. Numer. Algorithms 74, 927-935 (2017)

21. Wang, F.: Polyak's gradient method for split feasibility problem constrained by level sets. Numer. Algorithms 77, 925-938 (2018)

22. Xu, H.K.: Iterative algorithms for nonlinear operators. J. Lond. Math. Soc. 66, 240-256 (2002)

23. Xu, H.K.: A variable Krasnosel'skii-Mann algorithm and the multiple-set split feasibility problem. Inverse Probl. 22, 2021-2034 (2006)

24. Xu, H.K.: Iterative methods for the split feasibility problem in infinite-dimensional Hilbert spaces. Inverse Probl. 26 105018 (2010)

25. Xu, H.K.: Averaged mappings and the gradient-projection algorithm. J. Optim. Theory Appl. 150(2), 360-378 (2011)

26. Xu, H.K.: Properties and iterative methods for the Lasso and its variants. Chin. Ann. Math., Ser. B 35(3), 501-518 (2014)

27. Yang, Q: The relaxed CQ algorithm solving the split feasibility problem. Inverse Probl. 20, 1261-1266 (2004)

28. Yang, Q.: On variable-step relaxed projection algorithm for variational inequalities. J. Math. Anal. Appl. 302, 166-179 (2005)

29. Yao, Y., Liou, Y., Postolache, M.: Self-adaptive algorithms for the split problem of the demicontractive operators. Optimization 67(9), 1309-1319 (2018) 